\title{
CHALLENGE TO A STRAIGHT STRUCTURE FOR X-BAND LINEAR COLLIDER
}

\author{
K. Asano* , Y. Funahashi, Y. Higashi, T. Higo ${ }^{\#}$, N. Hitomi, T. Suzuki, K. Takata, \\ T. Takatomi, N. Toge, Y. Watanabe \\ KEK, High Energy Accelerator Research Organization, Oho 1-1, Tsukuba, Ibaraki, 305-0081, Japan
}

\begin{abstract}
The structure of the X-band main linac for the linear collider requires a stringent straightness of the order of several microns. Especially the misalignment from cell-to-cell to over twenty cells should be minimized. An one-shot diffusion bonding technology has been developed at KEK aiming at this requirement. Two $1.3 \mathrm{~m}$-long detuned structures have been made with a gentle bow of 20 microns. Recently the technology was applied to the main body bonding of an $1.8 \mathrm{~m}$-long damped-detuned structure, DDS3. It resulted in a much larger bow but the cell misalignment showed very smooth behavior and can later be corrected mechanically. The technique is being refined to obtain a better performance of the main body of the round DDS structure, RDDS1. In the present paper are described the studies on the basic techniques applied for DDS3.
\end{abstract}

\section{INTRODUCTION}

For preserving a very low emittance through a linac for linear collider in a multi-bunch operation, it is essential to reduce the emittance dilution due to the long-range transverse wake field. For this aim, the frequencies of the transverse modes in an accelerating structure should be well distributed as designed and also the position of those modes should be located transversely within several microns level.

Several detuned structures were fabricated up to now to study the feasibility of realizing these requirements in Xband disk-loaded structures[1]. From these experiences, it was found that the one-shot diffusion bonding technology for joining the precisely machined cells to form a solid structure of one-meter size was very promising to meet the above requirements[1,2]. These structures were $80 \mathrm{~mm}$ in diameter and $1.3 \mathrm{~m}$ in length. Frequency change due to bonding was small enough and the straightness were 10 to $20 \mu \mathrm{m}$, also satisfying the above requirements.

However, we decided to change the design of the structure to decrease the single-bunch wake field and to reduce the number of structures for a linac by enlarging the beam hole aperture and making the structure long. This decision was made in a collaborative work with SLAC[3]. The structure length became $1.8 \mathrm{~m}$. We also accepted the cell diameter of $61 \mathrm{~mm}$ as a working assumption.

Though it seems reasonable to choose the above mechanical parameters, the thin body makes the structure stiffness much lower than before. The geometrical moment of inertia of its cross section became small by a factor of 3. In addition, the long body makes its bow larger. If the structure is supported at both ends in a horizontal position, the sag due to self-weight becomes larger than before by a factor of 8 . Considering this dependence into account, it was estimated that the structure of the present design may suffer from a difficulty in obtaining a good straightness than before.

On the other hand, we have supplied the cells for two $1.8 \mathrm{~m}$-long DDS structures for SLAC with $61 \mathrm{~mm}$ in diameter. In the first step, each 38-cell stack is bonded followed by the second step, where several stacks are brazed into a structure. It suffered from kinks between 38cell stacks. In making the third structure, DDS3, we agreed to apply the same one-shot bonding method for its main body formation to realize a smoother variation of cell misalignment. This time LLNL made the cells where such a key characteristics as cell flatness of $0.5 \mu \mathrm{m}$, necessary for diffusion bonding, was surely realized [4].

In the present paper, we describe the studies and measurements done for stacking and bonding cells focusing on the cell misalignment.

\section{STUDY PROCEDURES}

One of the relevant issues of DDS3 is the straightness of the cells along the structure. A tolerance estimation[5] for the cell random misalignment along the structure shows about $5 \mu \mathrm{m} \mathrm{rms} \mathrm{misalignment} \mathrm{tolerance} \mathrm{in} \mathrm{a} \mathrm{regime} \mathrm{from}$ cell-to-cell to over than 10 cells. The tolerance for a longer wave length in misalignment relaxes as the wave length increases. Moreover, the global bow with a long wave length can easily be corrected through a mechanical measurement and forced bending before installation. Considering these in mind, we set targets for cell rms alignment of DDS3 as (1) cell-to-cell alignment to a few $\mu \mathrm{m}$ and (2) global one to $10 \mu \mathrm{m}$.

A dummy structure, DDS3D1, was firstly fabricated in order to confirm the method for DDS3. It was made directly following our previous technique, i.e. stacked on V-block, axially compressed at about $24 \mathrm{~kg}$, then rotated to vertical position to be one-shot diffusion bonded (DB) at a high temperature. Unfortunately a bad design of jigging caused a big failure. Then, we tried to find the

\footnotetext{
* Present address: Electrical Engineering Department, Akita National College of Technology, 1-1 Iijima-bunkyo-cho, Akita-shi 011-8511, Japan.

\# e-mail address: higo@mail.kek.jp
} 
method for obtaining a smooth structure with least possibility to have fatal errors again.

It was known already then through a pilot study of pre-bonding process (PB) that it could make a fairly smooth $1.8 \mathrm{~m}$-long structure with $61 \mathrm{~mm}$ in diameter[6]. In the method the cells are bonded with axially pressed by $600 \mathrm{~kg}$ force and kept in a furnace at $180{ }^{\circ} \mathrm{C}$ for a day. This makes the bonding between cells which prevents further mutual movement in the following process such as a final diffusion bonding.

We decided to take the advantage of this technique for DDS3. For investigating relevant parameters and getting experiences, we made another dummy structure, DDS3D2, before making DDS3. Following table shows the processes for those structures and some of results on misalignment of cells.

Table 1 List of processes for three structures.

\begin{tabular}{lccc}
\hline Structure & DDS3D1 & DDS3D2 & DDS3 \\
\hline Cell & Cylindrical & $3 \mathrm{D}$ & $3 \mathrm{D}$ \\
V block & Granite & $\mathrm{Cr} / \mathrm{Fe}$ & $\mathrm{Cr} / \mathrm{Fe}$ \\
1st Bonding & $\mathrm{DB}$ & $\mathrm{PB}$ & $\mathrm{PB}$ \\
2nd Bonding & None & $\mathrm{DB}$ & $\mathrm{DB}$ \\
Inclination & $<0.2 \mathrm{mrad}$ & $\sim 0.5 \mathrm{mrad}$ & $\sim 1 \mathrm{mrad}$ \\
Bow(P-V) & $\sim 10 \mathrm{~mm}$ & $0.5 \mathrm{~mm}$ & $0.23 \mathrm{~mm}$ \\
cell-to-cell(rms.) & - & - & $<1 \mu \mathrm{m}$ \\
\hline $\begin{array}{l}\text { DB=diffusion bonding at } 890{ }^{\circ} \mathrm{C}, \mathrm{PB}=\text { pre-bonding at } 180{ }^{\circ} \mathrm{C} \text { for two } \\
\text { days. }\end{array}$
\end{tabular}

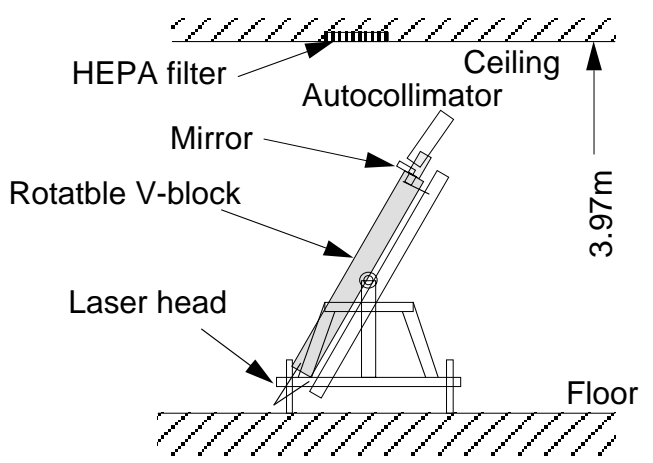

Fig. 1 Stacking set up in a clean room.

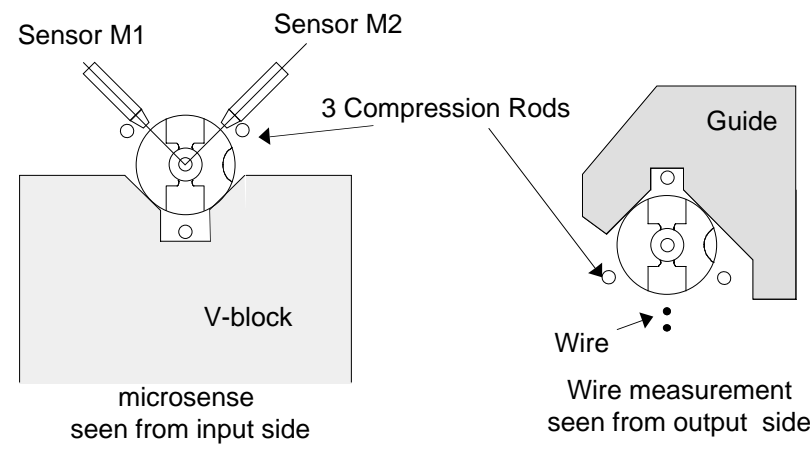

Fig. 2 Alignment measurement setup. Left: Capacitive sensors running along the V-block. Right: Vertically standing situation to measure the gap to a stretched wire.
A schematic drawing of the set up for stacking and measurement is shown in Fig. 1. The cells are stacked on a slanted V-block, typically at 60 degrees from horizontal, where the cell misalignment is also measured by running two capacitive gap sensors along the V-block as shown in Fig. 2(Left). The stacked cells and bonded ones are also measured in a vertical position by measuring the gap between the cells and a vertically stretched wire. These configurations are shown in Fig. 2(Right).

\section{RESULTS}

The quality of cells in three structures were similar; i.e. flatness of cells were mostly less than $0.5 \mu \mathrm{m}$ as shown in Fig. 3.

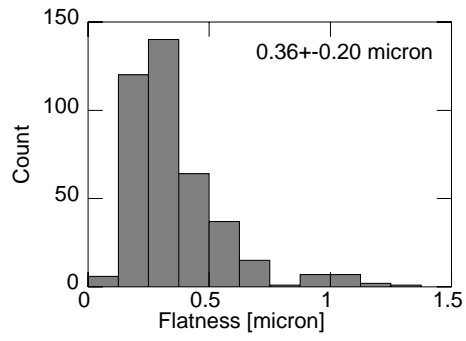

Fig. 3 Flatness of DDS3 cells. Average value and standard deviation are 0.36 and $0.20 \mu \mathrm{m}$, respectively.

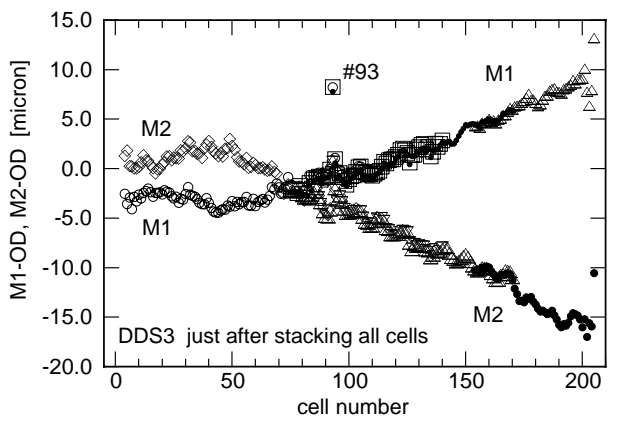

Fig. 4 Typical alignment of cells of DDS3 after stacking. Transverse constraint was applied during measurement.

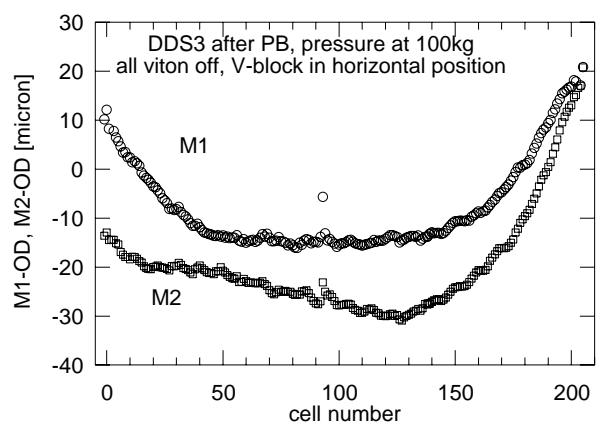

Fig. 5 Misalignment of cells after pre-bondingof DDS3.

Fig. 4 shows a typical example of the cell alignment of DDS3 just after stacking on a slanted V-block. A constraint against the transverse motion of cells was applied by Viton plates backedby stainless plates. In this case, the stacked-cell pillar was stable even if axial force of $100 \mathrm{~kg}$ was applied. 
However, after pre-bonding process, the alignment of the structure became as shown in Fig. 5, where the alignment pattern changes by a few tens of microns. This measurement was performed by reducing the axial force to $100 \mathrm{~kg}$. After diffusion bonding, the alignment became as shown in Fig. 6, where the structure was supported at two Bessel points without any axial force. Much larger global bow of about $230 \mu \mathrm{m}$ was found to be in a plane, almost horizontal direction w.r.t. V-block.

The bow is huge but the random cell-to-cell alignment is less than 1 micron (rms) as shown in Fig. 7, where the slowly varying part from Fig. 6 was subtracted through a third-order polynomial fitting.

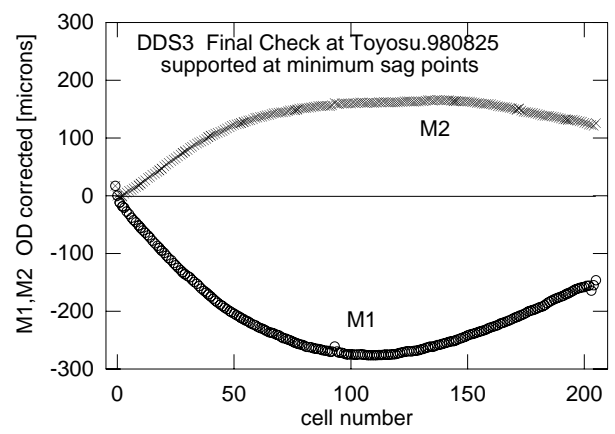

Fig. 6 Misalignment of cells after diffusion bonding of DDS3.

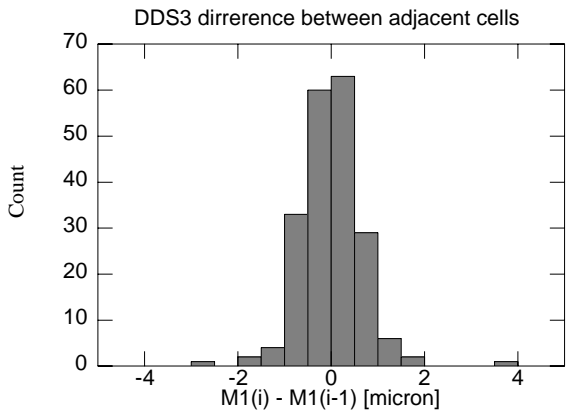

Fig. 7 Distribution of random cell-to-cell misalignment of DDS3 cells in a direction of one of the sensors.

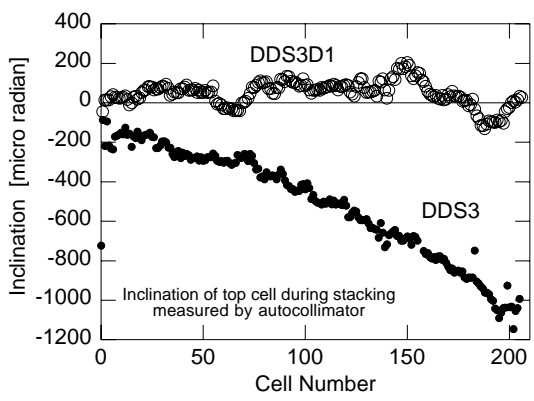

Fig. 8 Inclination of the lastly-stacked cell on top. Negative values mean falling down towards V-block.

An important issue was discussed after SLAC's measurement of a big bookshelf of the order of $500 \mu \mathrm{rad}$ in DDS3[7]. The observed bookshelf seems consistent within a factor of two to the measured inclination of the top cell while stacking shown in Fig. 8. It is to be noted that the inclination in the case of DDS3D1 did not show the similar pattern, while it did in the DDS3D2 case, indicating the cause from the different processes before bonding. The relevant differences between the two cases are such as material of V-block and cylindrically symmetry of the cells, which should be studied in future.

\section{SUMMARY AND DISCUSSION}

Much experiencewas obtained through the three structure fabrications and these are feedback to the studies towards an on-going program, RDDS1.

The DDS3 main body bonding was successfully done with applying the pre-bonding process followed by a high-temperature diffusion bonding. The structure became smoothly bent with a maximum of $230 \mu \mathrm{m}$ in a plane but the random cell-to-cell slippage was less than $1 \mu \mathrm{m}$ (rms). These features satisfy the requiredcell alignment tolerance because mechanical straightening can be performed. However such a straightening by a factor more than 10 is probably too much so that we should develop a method to make a structure with a bow at most several tens of microns.

The bookshelving became as large as 1 mrad in DDS3. Mechanism and cures should be studied to suppress it down to a tolerable level of $50 \mu \mathrm{rad}$.

Now we are preparing the RDDS1 following basically the same technology. So it may refrain the same bad features but the fabrication is planned to proceed more carefully such as by (1) checking the inclination in both directions, (2) checking bookshelf not only at the top cell but also at the alreadystacked cells, (3) stacking cells on a more stable base, (4) pressing axially with less transverse component and (5) making a better transverse constraint.

\section{ACKNOWLEDGMENTS}

This program was proceeded in a collaboration among three laboratories, Stanford Linear Accelerator Center (SLAC), Lawrence Livermore National Laboratory (LLNL) and High Energy Accelerator Research Organization (KEK). The authors greatly acknowledge those who support this collaboration.

All processes were done at Ishikawajima-Harima Heavy Industries (IHI) directedby KEK. It was proceeded as one of the contracts, but the authors want to express much thank to those staffs of the company for making great efforts to successfully complete this series of jobs.

\section{REFERENCES}

[1] T. Higo et al., Proceedings of The First Asian Particle Accelerator Conference, APAC98, Tsukuba, Japan.

[2] JLC Design Study, KEK Report 97-1, 1997.

[3] T. Raubenheimer and K. Yokoya, LCC-0003, SLAC, 1998.

[4] J. Klingmann et al., in this conference, MOP51, PAC99, 1999.

[5] G. Stupakov, presentation at ISG3, SLAC, Jan. 1999.

[6] N. Hitomi, presented at ISG1, SLAC, Jan. 1998, LCC-0002

[7] C. Pearson, presented at ISG3, SLAC, Jan. 1999. 